\title{
Long-Term Clinical Results of Unicompartmental Knee Arthroplasty in Patients Younger than 60 Years of Age: Minimum 10-Year Follow-up
}

\author{
Kyung Tae Kim, MD, Song Lee, MD, Jung Soo Lee, MD, Min Su Kang, MD, and Ki Hyuk Koo, MD \\ Department of Orthopedic Surgery, Seoul Sacred Heart General Hospital, Seoul, Korea
}

\begin{abstract}
Purpose: To evaluate the long-term clinical results and survivorship of unicompartmental knee arthroplasty (UKA) in patients younger than 60 years of age.

Materials and Methods: One hundred and six cases of medial UKA with a minimum follow-up of 10 years were selected for this study. There were 80 patients and the preoperative diagnosis was osteoarthritis in all cases. The mean age of the patients was 54.2 years and the mean duration of followup was 12.1 years. Clinical assessments were performed using the Knee Society clinical rating system, and a survival analysis was performed using the Kaplan-Meier method.

Results: The mean knee and function scores improved from $52.8 \pm 8.4$ points and $56.6 \pm 10.6$ points preoperatively to $85.4 \pm 9.1$ points and $84.7 \pm 10.4$ points at the last follow-up, respectively $(\mathrm{p}<0.001)$. The mean range of motion was recovered from $130.7^{\circ}$ to $132.8^{\circ}$ at the last follow-up. Complications occurred in 20 cases (16.7\%) and the most prevalent complication was mobile bearing dislocation ( $\mathrm{n}=9,7.5 \%)$. The 10 -year survival rate was $92.8 \%$ when conversion to total knee arthroplasty was defined as failure, whereas $89.3 \%$ when failure was defined as all revision surgeries.

Conclusions: The long-term clinical results of UKA were satisfactory in patients under 60 years of age. Therefore, UKA could be a useful method for the treatment of medial compartment osteoarthritis of the knee in patients younger than 60 years of age.
\end{abstract}

Keywords: Knee, Osteoarthritis, Arthroplasty, Unicompartmental, Survivorship, Younger patient

\section{Introduction}

Unicompartmental knee arthroplasty (UKA) was primarily performed in patients over 60 years of age in the past. Its indications have since been expanded based on promising results in various recent studies. Accordingly, the incidence of UKA is also increasing in relatively young less than 60 -year-old patients ${ }^{1-6)}$. However, UKA in young patients is a challenging procedure due to their

Received April 27, 2017; Revised (1st) June 27, 2017;

(2nd) August 12, 2017; (3rd) August 25, 2017; Accepted August 28, 2017

Correspondence to: Kyung Tae Kim, MD

Department of Orthopedic Surgery, Seoul Sacred Heart General Hospital, 259 Wangsan-ro, Dongdaemoon-gu, Seoul 02488, Korea Tel: +82-2-966-1616, Fax: +82-2-968-2394

E-mail: kktkimos@hanmail.net

This is an Open Access article distributed under the terms of the Creative Commons Attribution Non-Commercial License (http://creativecommons.org/licenses/by-nc/4.0/) which permits unrestricted non-commercial use, distribution, and reproduction in any medium, provided the original work is properly cited. high expectation on postoperative knee function and long life expectancy, and there is a concern that they are exposed to a higher risk of revision ${ }^{3,6,7)}$.

There is a broad consensus that UKA in less than 60-yearold patients offers good clinical outcome in terms of pain relief and improvement of knee and function scores, and recovery of range of motion (ROM); however, there is still controversy over the long-term survivorship of the implant ${ }^{6-9)}$. A relatively small number of studies have been published on the long-term results of UKA in young patients. In addition, survivorship studies with only the cases of more than 10-year follow-up are almost nonexistent. Therefore, the purpose of this study is to investigate the long-term survivorship and functional outcome of UKA in less than 60-year-old patients based on minimum 10-year follow-up clinical data. Our hypothesis is that UKA provides good longterm clinical outcome in relatively young patients. 


\section{Materials and Methods}

A total of 120 cases of UKA were performed for 2 years between January 2002 and December 2003 on 93 patients younger than 60 years of age. Of those, 106 cases (80 patients) were available for more than 10 years of follow-up, excluding 14 cases ( 1 due to death and 13 due to loss to follow-up). All patients were female with a mean age of 54.2 years (range, 45 to 59 years). The mean follow-up was 12.1 years (range, 10 to 13 years). The preoperative diagnosis was degenerative osteoarthritis in all cases.

The surgery was indicated for patients with medial compartment pain and limited mobility without any inflammatory knee joint disease if the anterior and posterior cruciate ligaments were functionally intact and varus deformity and flexion contracture was $\leq 15^{\circ}$ and $\mathrm{ROM}$ was $\geq 110^{\circ}$. UKA was performed on patients with radiographic evidence of osteophyte formation without narrowing of the lateral joint space and patellofemoral joint space and those without patellofemoral joint pain in the presence of degenerative osteoarthritis. The exclusion criteria included preoperative findings of anterior/posterior instability, degenerative changes such as joint space narrowing in the lateral compartment, and anterior knee pain during level-ground walking or stair climbing in patients with patellofemoral joint osteoarthritis.

The surgery was performed at a single institution in all cases by two surgeons using a minimally invasive surgical technique. The Oxford phase 3 (Biomet, Warsaw, IN, USA) mobile bearing knee prosthesis was implanted in all knees (Fig. 1). A short skin incision was made on the medial side of the patella from the su- perior border of the patella to inferior to the joint line. The joint was exposed via a medial parapatellar capsulotomy, and after checking the structures inside the joint, osteophyte removal was performed. Using an extramedullary tibial resection guide, proximal tibial resection was performed. The posterior condyle of the femur was resected using a femoral drill guide and a femoral cutting block. After flexion and extension gaps were measured using a feeler gauges, they were equalized by milling of the distal femoral condyle. The tibial and femoral components were fixed with bone cement, and a mobile polyethylene bearing was inserted between them.

Preoperative data were prospectively collected and regular follow-up was performed to assess clinical outcome. Clinical and radiographic assessments were performed through regularly scheduled follow-up at 3 months, 6 months, and 12 months after surgery and yearly thereafter. A phone interview was conducted for patients who were not available for a follow-up visit and their status was examined in later follow-up visits. On the clinical assessment, knee joint pain and ROM were assessed and knee score and function score were calculated using the Knee Society clinical rating system. Overall results according to knee score were classified into 4 categories (100-85 points, excellent; $84-70$ points, good; 69-60 points, fair; and $\leq 59$ points, poor ${ }^{10)}$. On the radiographic assessment, the tibiofemoral angle was measured and the presence of bony change, component loosening/wear, and dislocation of components were examined on the weight bearing radiographs of the knee. Patients who underwent further surgery due to postoperative complications were divided into two groups
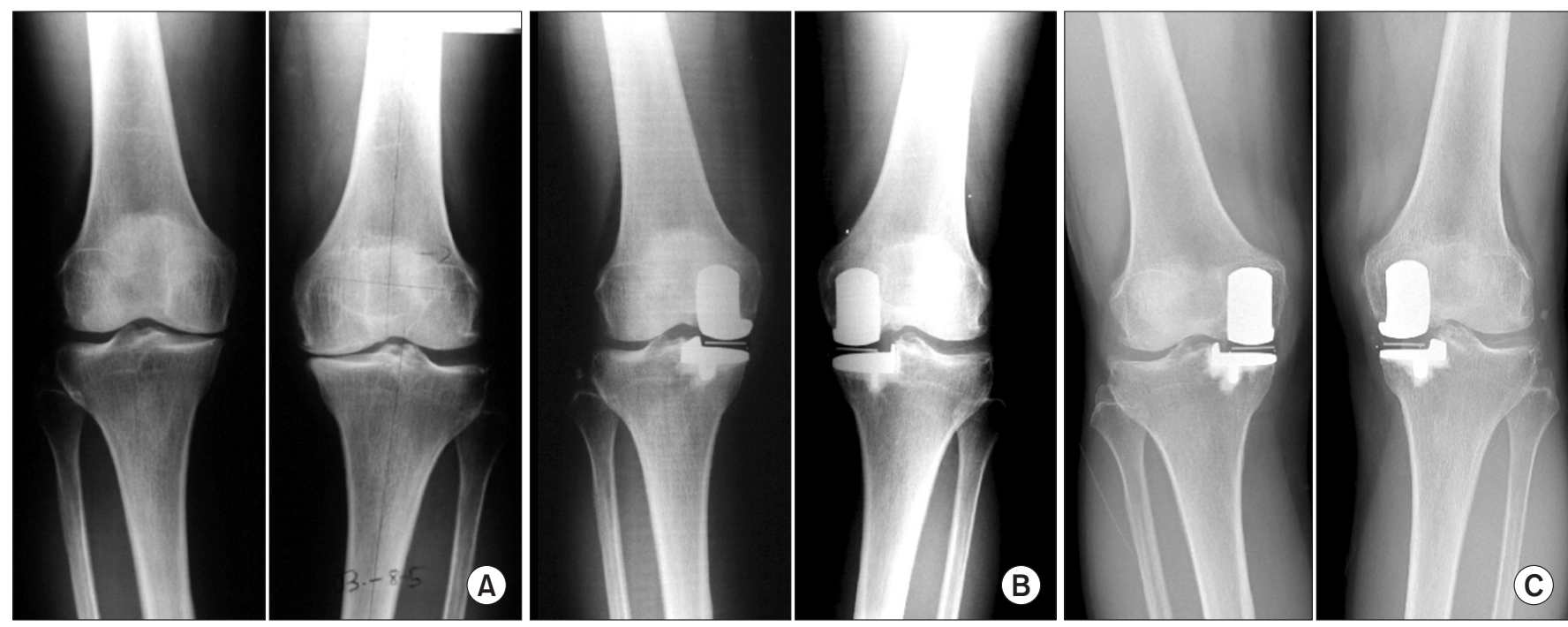

Fig. 1. (A) Preoperative radiographs of a 57-year-old female show osteoarthritis of the medial compartment in both knees. (B) Postoperative radiographs of minimally invasive Oxford phase 3 (Biomet) unicompartmental knee arthroplasty (UKA). (C) Last follow-up radiographs obtained at 13 years after UKA. 
(revision group vs. reoperation other than revision group). Failure of the index surgery was defined as exchange/removal of any component. For statistical analysis, a paired $t$-test or Wilcoxon signed-rank test was used depending on the normality of data distribution. A p $<0.05$ was considered statistically significant. Survival analysis was estimated using the Kaplan-Meier method including the death and lost-to follow-up as censored with the 95\% confidence interval (CI).

\section{Results}

\section{Clinical Outcome}

The mean Knee Society knee score was significantly improved from 52.8 points (range, 25 to 67 points) preoperatively to 85.4 points (range, 60 to 100 points) at the last follow-up ( $\mathrm{p}<0.001)$. The Knee Society knee function score was also significantly improved from 56.6 points (range, 25 to 80 points) preoperatively to 84.7 points (range, 60 to 100 points) at the last follow-up ( $\mathrm{p}<0.001$ ). The mean $\mathrm{ROM}$ was restored from $130.7^{\circ}$ (range, $110^{\circ}$ to $135^{\circ}$ ) preoperatively to $132.8^{\circ}$ (range, $105^{\circ}$ to $135^{\circ}$ ) at the last follow-up $(\mathrm{p}<0.05)$. The mean tibiofemoral angle was changed

Table 1. Clinical Results

\begin{tabular}{lccc}
\hline & Preoperative & Last follow-up & p-value \\
\hline Knee Society score & $52.8 \pm 8.4$ & $85.4 \pm 9.1$ & $0.000^{\text {a) }}$ \\
Knee score & $56.6 \pm 10.6$ & $84.7 \pm 10.4$ & $0.000^{\text {b) }}$ \\
Function score & $130.7 \pm 9.3$ & $132.8 \pm 5.7$ & $0.045^{\text {b) }}$ \\
Range of knee motion $\left(^{\circ}\right)$ & $-1.2 \pm 3.1$ & $4.7 \pm 2.9$ & $0.000^{\text {b) }}$ \\
Tibiofemoral angle $\left(^{\circ}\right)$ & & &
\end{tabular}

Values are presented as mean \pm standard deviation.

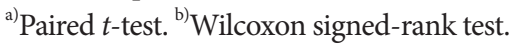

from $1.2^{\circ}$ varus (range, $8^{\circ}$ varus to $6^{\circ}$ valgus) preoperatively to $4.7^{\circ}$ valgus (range, $2^{\circ}$ varus to $10^{\circ}$ valgus) at the last follow-up $(\mathrm{p}<0.001)$ (Table 1). According to the Knee Society clinical rating system, 63 cases (69.2\%) were classified as excellent, 24 cases (26.4\%) as good, and 4 cases (4.4\%) as fair by Insall's criteria (Table 2).

\section{Complications}

Postoperative complications occurred in 20 cases (16.7\%). Mobile bearing dislocation ( 9 cases, $7.5 \%$ ) was the most prevalent complication, which was followed by implant loosening (3 cases, $2.5 \%$ ), polyethylene wear ( 2 cases), mobile bearing impingement against the osteophyte ( 2 cases), periprosthetic fracture (2 cases), medial collateral ligament injury (1 case), and lateral compartment osteoarthritis (1 case). There was no case of infection. Reoperation other than revision knee arthroplasty was required in 5 cases $(4.2 \%)$ at a mean of 1.2 years after surgery (range, 0 to 3.2 years). Revision knee arthroplasty was performed in 15 cases $(12.5 \%)$ at a mean of 6.0 years after surgery (range, 0.3 to 11.8 years) (Table 3 ).

\section{Survivorship}

The 10-year cumulative survivorship was 92.8\% (95\% CI, 88.1

Table 2. Overall Results according to Knee Society Knee Scores

\begin{tabular}{lcc}
\hline \multicolumn{1}{c}{ Score } & Preoperative & Last follow-up \\
\hline Excellent $(85-100)$ & $0(0)$ & $63(69.2)$ \\
Good $(70-84)$ & $0(0)$ & $24(26.4)$ \\
Fair $(60-69)$ & $29(31.8)$ & $4(4.4)$ \\
Poor $(\leq 59)$ & $62(68.2)$ & $0(0)$
\end{tabular}

Values are presented as number (\%).

Table 3. Complications of Unicompartmental Knee Arthroplasty

\begin{tabular}{lccl}
\hline \multicolumn{1}{c}{ Complication } & No. of cases $(\%)$ & Time interval (mo) & \multicolumn{1}{c}{ Treatment } \\
\hline Bearing dislocation & $9(7.5)$ & $62.3 \pm 40.2$ & 6 revision TKA, 3 bearing change \\
Implant loosening & $3(2.5)$ & $119 \pm 61.5$ & 3 revision TKA \\
Polyethylene wear & $2(1.7)$ & $85 \pm 4.2$ & 2 bearing change \\
Periprosthetic fixation & $2(1.7)$ & $19 \pm 26.8$ & 1 OR/IF, 1 CR/IF \\
Impingement & $2(1.7)$ & $15 \pm 8.5$ & 2 arthroscopy \\
Contralateral OA & $1(0.8)$ & 140 & 1 revision TKA \\
MCL injury & $1(0.8)$ & 3 & 1 MCL repair \\
Total & $20(16.7)$ & $62.6 \pm 51.4$ & 10 revision TKA, 5 bearing change, 2 arthroscopy, \\
\end{tabular}

Values are presented as mean \pm standard deviation.

TKA: total knee arthroplasty, OR/IF: open reduction/internal fixation, CR/IF: closed reduction/internal fixation, OA: osteoarthritis, MCL: medial collateral ligament. 


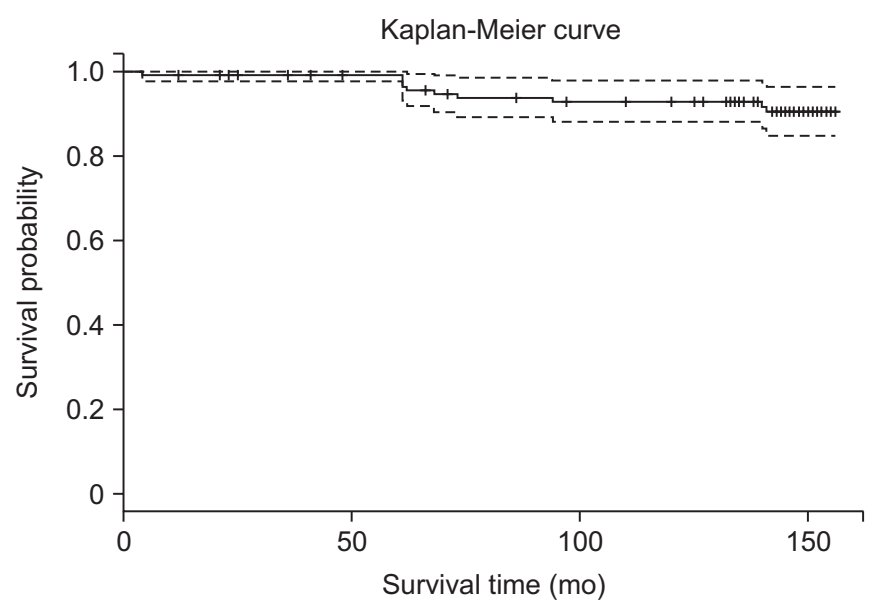

Fig. 2. Kaplan-Meier survivorship analysis curve showing the survival rate of unicompartmental knee arthroplasty of 92.8\% (95\% confidence interval [CI], 88.1 to 97.7 ) at 10 years and $90.4 \%$ (95\% CI, 84.9 to 96.3 ) at 13 years with conversion to total knee arthroplasty as the end point. +: censored.

to 97.7 ), and the 13-year survivorship was $90.4 \%$ (95\% CI, 84.9 to 96.3) when conversion to total knee arthroplasty (excluding simple bearing change) was defined as failure (Fig. 2). When all revision surgeries including mobile bearing change were defined as failure, the 10-year cumulative survivorship was $89.3 \%$ (95\% CI, 83.7 to 95.2$)$ and the 13 -year survivorship was $85.8 \%$ (95\% CI, 79.3 to 92.8 ) (Fig. 3).

\section{Discussion}

In this study, we evaluated the long-term follow-up results of UKA in less than 60-year-old patients who underwent the procedure for degenerative osteoarthritis. The minimum 10-year follow-up (mean, 12.1 years) results showed excellent knee function and satisfactory implant survivorship in this patient population. The results confirmed our hypothesis that UKA would offer good long-term results in less than 60 -year-old patients. Therefore, we believe that UKA can be an effective procedure for the treatment of degenerative osteoarthritis in relative young patients.

Treatment of degenerative osteoarthritis of the knee in young patients has been considered challenging, and it has been the subject of debate ${ }^{11-14)}$. Non-surgical treatment may be effective for advanced osteoarthritis in terms of pain relief and functional improvement; however, surgical procedures, such as high tibial osteotomy, UKA, and total knee arthroplasty should be considered for unrelenting symptoms ${ }^{9,11,14,15)}$. In particular, UKA has been recommended for relatively less active advanced age patients $(\geq 60$ years of age) with unicompartmental osteoarthritis. Recently,

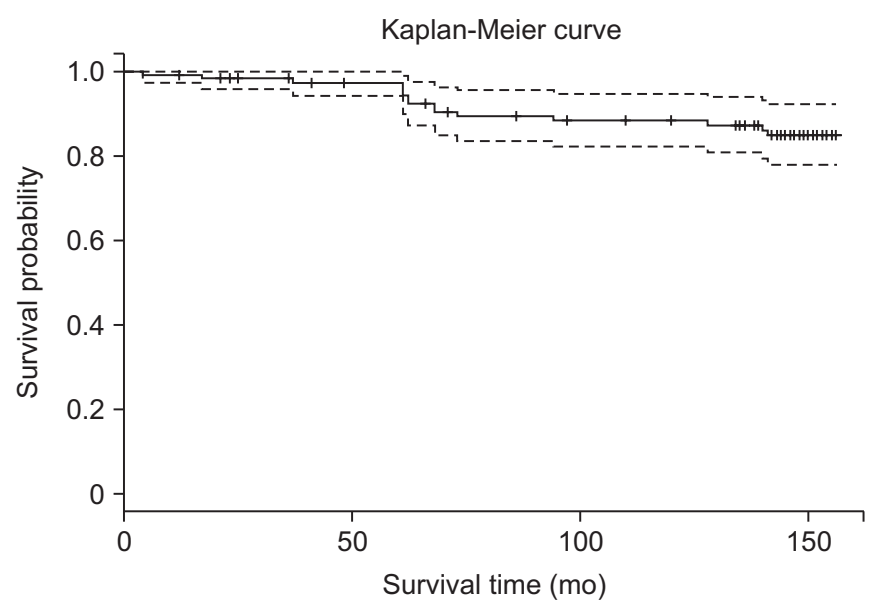

Fig. 3. Kaplan-Meier survivorship analysis curve showing the survival rate of unicompartmental knee arthroplasty of $89.3 \%$ (95\% confidence interval [CI], 83.7 to 95.2$)$ at 10 years and $85.8 \%(95 \%$ CI, 79.3 to 92.8$)$ at 13 years with failure for any reason as the end point. +: censored.

however, with use of minimally invasive techniques requiring a small incision and other improved surgical techniques and instruments, a growing body of studies has demonstrated favorable outcomes of UKA, which has led to an increase in the incidence of UKA in relative young patients ( $<60$ years of age).

Several recent studies have shown clinical outcome of UKA comparable to those of total knee arthroplasty in less than 60-year-old patients ${ }^{1,14,16-18)}$. In a study by Pennington et al. ${ }^{6}$, UKA using fixed bearing Miller-Galante prostheses resulted in excellent outcomes in $93 \%$ of the less than 60 -year-old patients: the mean Hospital for Special Surgery knee score was 94 points and the mean UCLA activity score was 6.5 points at a mean of 11-year follow-up. Bruni et al. ${ }^{11)}$ followed 33 cases of all-poly tibial component UKA for a mean of 8 postoperative years. Less than 60-year-old patients in the study obtained significant improvement after UKA with a mean Knee Society knee score of 87 points, a mean Knee Society function score of 84 points, a mean Western Ontario and McMaster Universities Osteoarthritis Index (WOMAC) score of 85 points, and a mean ROM of $125^{\circ}$. Kort et al. ${ }^{3)}$ obtained excellent clinical outcomes of Oxford phase 3 mobile bearing UKA in all of their less than 60-year-old patients. At 2 to 6 years after surgery, the mean Knee Society knee score was 90.5 points, the mean Knee Society function score was 89.4 points, the mean WOMAC pain score was 76 points, the mean WOMAC function score was 73 points, and the ROM was $125^{\circ}$. Walker et al. ${ }^{1)}$ observed most of their patients were satisfied at a mean of 4.4 years after mobile bearing UKA and returned to activities of daily living. In addition, $2 / 3$ of the patients achieved a high activity level (UCLA score $\geq 7$ points). In a more recent re- 
port, Faour Martin et al. ${ }^{12)}$ reported excellent or good results were obtained in 53 (96.4\%) of total 55 cases of UKA performed in less than 60-year-old patients: at a mean of 12-year follow-up, the mean American Knee Society (AKS) knee score was 92 points, the mean AKS function score was 89 points, and the mean ROM was $134^{\circ}$. In 2017 , Streit et al. ${ }^{14)}$ reported that high satisfaction and excellent clinical results of their $\leq 60$-year-old patients at a mean of 5 years after surgery using Oxford mobile bearing UKA: the AKS score was 89 points, mean ROM was $129^{\circ}$, and the mean UCLA score was 6.8 points. In the current study, we found significant improvement in our less than 60-year-old patients after Oxford phase 3 mobile bearing UKA. At a mean followup of 12.1 years, the postoperative Knee Society knee score and function score were 85.4 points and 84.7 points, respectively, and the mean ROM was improved to $132.8^{\circ}$. Thus, excellent or good results were achieved in $95.6 \%$ of the total patients.

On the survivorship of fixed bearing UKA in less than 60-yearold patients, Parratte et al. ${ }^{13)}$ reported that the 12-year survivorship of Miller-Galante metal-backed prosthesis $(n=35)$ was $80.6 \%$ in $\leq 50$-year-old patients. In a study by Pennington et al. ${ }^{6}$, the 11 year survivorship was $92 \%$ in $\leq 60$-year-old patients after MillerGalante fixed bearing UKA. Heyse et al. ${ }^{8)}$ reported the 10-year survivorship of Genesis fixed bearing UKA as $94.1 \%$ in patients $<60$ years. On the survivorship of mobile bearing UKA, Price et al. ${ }^{9)}$ found no significant difference between patients $<60$ years and $\geq 60$ years with the 10-year survivorship in the former group (52 cases) being 91\% after Oxford mobile bearing UKA. Recent studies on Oxford mobile bearing UKA have shown excellent survival rates: 97\% 5-year survivorship in $\leq 60$ years $(118 \text { cases })^{14)}$ and $95 \% 12$-year survivorship in $\leq 59$ years $(55 \text { cases })^{12)}$. However, $\mathrm{W}$-Dahl et al. ${ }^{7)}$ reported that the 7-year cumulative risk of revision data from the Australian and Swedish knee registries showed that patients aged less than 65 years had significantly higher rates of revision than those in 65 years and older ( $<55$ years, 19\%; 55 to 64 years, $13 \%$; 65 to 74 years, $8.6 \%$; and $\geq 75$ years, $5.7 \%$ ). Thus, they suggested that surgeons should be aware of the higher risk of revision after UKA in young patients. In the current study, revision knee arthroplasty was performed in 15 of 106 cases. When failure was defined as revision knee arthroplasy including mobile bearing change, the 10-year cumulative survivorship was $89.3 \%$ and the 13 -year cumulative survivorship was $85.8 \%$.

Studies that compare two different age groups directly after UKA are rare. Thompson et al. ${ }^{17)}$ reported that the mean Knee Society knee score at 2 years after fixed bearing UKA was significantly higher in patients younger than 60 years old than patients 60 years and older (93.3 vs. 77.7 ), which they attributed to the improved modern implant design and surgical technique. Pandit et al. ${ }^{18)}$ compared the less than 60-year-old patients (245 cases) and those 60 years and older ( 755 cases) at a mean of 5.6 years after mobile bearing UKA. The Oxford knee score and AKS score were not significantly different between groups, whereas the AKS function score and Tegner activity score were higher in patients younger than 60 years. There was no significant difference in the 10-year survivorship between the younger patients (97.3\%) and the older patients (95.1\%).

Possible complications of UK include polyethylene wear, dislocation of mobile bearing, aseptic loosening, arthritis in the other compartment, infection, periprosthetic fracture, limited ROM, and unexplained severe pain. Due to the higher level of activity, younger patients have been thought to be more vulnerable to complications such as component wear and loosening ${ }^{3,13)}$. In the study by Pandit et al. ${ }^{18)}$, complications occurred in $6(2.5 \%)$ out of 245 cases in the younger than 60 year group and dislocation of the mobile bearing ( 3 cases) was most common. In the 60 year and over group, 23 (3.0\%) out of 755 cases had complications, of which progression of arthritis to the other compartment (7 cases) was most common. Parratte et al. ${ }^{13)}$ reported that polyethylene wear was the most prevalent complication in less than 50-yearold patients: complications developed in 6 out of 35 cases after fixed bearing UKA, and there were 4 cases of polyethylene wear, 1 case of component loosening, and 1 case of progression of arthritis. In the current study, postoperative complications were found in $16.7 \%$ of the cases. Dislocation of the mobile bearing was most common (7.5\%), followed by component loosening (2.5\%). There were 2 cases (1.7\%) of polyethylene wear and 1 case $(0.8 \%)$ of lateral compartment osteoarthritis. There was no case of infection. Reoperations other than revision knee arthroplasty were performed in 5 cases (4.2\%) and revision knee arthroplasty was required in 15 cases $(12.5 \%)$.

The limitations of this study include that the 12.1 years of mean follow-up period was not enough to determine the final longterm outcome of UKA in less than 60-year-old patients and the study population consisted of female only. In addition, direct comparison with patients 60 years and over was not performed. However, the significance of this study lies in the fact that the results were based on all the cases were less than 60-year-old patients who were followed up for a minimum of 10 years, and it was a single center study involving a relatively large study population. 


\section{Conclusions}

The minimum 10-year long-term follow-up results of UKA performed in patients under 60 years of age were good in terms of Knee Society knee score and function score and ROM, and the survivorship was satisfactory. Therefore, UKA can be a useful method for the treatment of medial compartment osteoarthritis of the knee in patients younger than 60 years of age.

\section{Conflict of Interest}

No potential conflict of interest relevant to this article was reported.

\section{References}

1. Walker T, Streit J, Gotterbarm T, Bruckner T, Merle C, Streit MR. Sports, physical activity and patient-reported outcomes after medial unicompartmental knee arthroplasty in young patients. J Arthroplasty. 2015;30:1911-6.

2. Kim KT, Lee S, Ko DO, Cho KH, Kim KS, Kim TW. Comparative study for the results of unicompartmental knee arthroplasty in patients younger and older than 60 years of age. J Korean Orthop Assoc. 2009;44:192-200.

3. Kort NP, van Raay JJ, van Horn JJ. The Oxford phase III unicompartmental knee replacement in patients less than 60 years of age. Knee Surg Sports Traumatol Arthrosc. 2007;15: 356-60.

4. Kozinn SC, Scott R. Unicondylar knee arthroplasty. J Bone Joint Surg Am. 1989;71:145-50.

5. Murray DW. Mobile bearing unicompartmental knee replacement. Orthopedics. 2005;28:985-7.

6. Pennington DW, Swienckowski JJ, Lutes WB, Drake GN. Unicompartmental knee arthroplasty in patients sixty years of age or younger. J Bone Joint Surg Am. 2003;85:1968-73.

7. W-Dahl A, Robertsson O, Lidgren L, Miller L, Davidson D, Graves S. Unicompartmental knee arthroplasty in patients aged less than 65. Acta Orthop. 2010;81:90-4.

8. Heyse TJ, Khefacha A, Peersman G, Cartier P. Survivorship of UKA in the middle-aged. Knee. 2012;19:585-91.

9. Price AJ, Dodd CA, Svard UG, Murray DW. Oxford medial unicompartmental knee arthroplasty in patients younger and older than 60 years of age. J Bone Joint Surg Br. 2005;87: 1488-92.

10. Insall JN, Dorr LD, Scott RD, Scott WN. Rationale of the Knee Society clinical rating system. Clin Orthop Relat Res. 1989;(248):13-4.

11. Bruni D, Akkawi I, Iacono F, Raspugli GF, Gagliardi M, Nitri M, Grassi A, Zaffagnini S, Bignozzi S, Marcacci M. Minimum thickness of all-poly tibial component unicompartmental knee arthroplasty in patients younger than 60 years does not increase revision rate for aseptic loosening. Knee Surg Sports Traumatol Arthrosc. 2013;21:2462-7.

12. Faour Martin O, Valverde Garcia JA, Martin Ferrero MA, Vega Castrillo A, Zuil Acosta P, Suarez De Puga CC. The young patient and the medial unicompartmental knee replacement. Acta Orthop Belg. 2015;81:283-8.

13. Parratte S, Argenson JN, Pearce O, Pauly V, Auquier P, Aubaniac JM. Medial unicompartmental knee replacement in the under-50s. J Bone Joint Surg Br. 2009;91:351-6.

14. Streit MR, Streit J, Walker T, Bruckner T, Philippe Kretzer J, Ewerbeck V, Merle C, Aldinger PR, Gotterbarm T. Minimally invasive Oxford medial unicompartmental knee arthroplasty in young patients. Knee Surg Sports Traumatol Arthrosc. 2017;25:660-8.

15. Hanssen AD, Stuart MJ, Scott RD, Scuderi GR. Surgical options for the middle-aged patient with osteoarthritis of the knee joint. Instr Course Lect. 2001;50:499-511.

16. Kim YJ, Kim BH, Yoo SH, Kang SW, Kwack CH, Song MH. Mid-term results of oxford medial unicompartmental knee arthroplasty in young asian patients less than 60 years of age: a minimum 5-year follow-up. Knee Surg Relat Res. 2017;29: 122-8.

17. Thompson SA, Liabaud B, Nellans KW, Geller JA. Factors associated with poor outcomes following unicompartmental knee arthroplasty: redefining the "classic" indications for surgery. J Arthroplasty. 2013;28:1561-4.

18. Pandit H, Jenkins C, Gill HS, Smith G, Price AJ, Dodd CA, Murray DW. Unnecessary contraindications for mobilebearing unicompartmental knee replacement. J Bone Joint Surg Br. 2011;93:622-8. 